\title{
Gold Nanoparticle Based Plasmonic Microwave-antenna
}

\author{
Ngo Hai Yen ${ }^{1}$, Dang Thi Thanh Thuy ${ }^{1,}$, , Nguyen Khac Thuan ${ }^{2}$ \\ ${ }^{1}$ Faculty of Physics, VNU-Hanoi University of Science, Ha Noi, Viet Nam \\ ${ }^{2}$ Faculty of Engineering Physics and Nanotechnology, VNU-University of Engineering and Technology, Ha Noi, Viet Nam
}

\section{Email address:}

dangthuyhn@gmail.com (D. T. T. Thuy)

${ }^{*}$ Corresponding author

\section{To cite this article:}

Ngo Hai Yen, Dang Thi Thanh Thuy, Nguyen Khac Thuan. Gold Nanoparticle Based Plasmonic Microwave-antenna. American Journal of Applied Scientific Research. Vol. 2, No. 6, 2016, pp. 82-86. doi: 10.11648/j.ajasr.20160206.18

Received: September 19, 2016; Accepted: November 29, 2016; Published: December 27, 2016

\begin{abstract}
In recent years, wireless communication has been developed towards the ultra-high bands in order to exploit the plasmonic effect that was observed in nanoscale metallic nanomaterials. In this report we study the impact of plasmon effect of gold nanoparticles covered on the surface of the microwave antenna to seek for a change in the antenna efficiency. In particular, the changes of antenna's reponses when exposing to the light and the dark were investigated. The obtained results show that the reponse loss (S1 coefficient) decreased considerably while preserving the position of the resonance lines.
\end{abstract}

Keywords: Antenna, Gold, Nanoparticles, Plasmon, Microwave

\section{Introduction}

The plasmonic properties of nanoparticles have attracted much attention in the past decades [1], [9], [10]. The noble metal nanoparticles strongly absorb visible light with efficiency estimated of around 95\% [7], [10], [11]. The color and therefore the spectrum of absorbed light depend on the shape and size of the nanoparticles which appeared to associate with the dielectric constants of surrounding medium. There are two modes of plasmonic effect: the volume and the surface plasmon. Whereas the volume plasmon originates in a collective oscillation of free electrons inside the bulk materials, the surface plasmon associates with the resonant oscillation of conduction electrons at the interfaces between a negative and positive permittivity material stimulated by incident light. The electromagnetic waves propagating along the dielectric-to-metal interface to form a small surface plasmon is usually called a surface plasmon polariton (SPP). The term "surface plasmon polariton" explains that the wave involves both charge motion in the metal and electromagnetic waves in the air or dielectric. The electromagnetic field of the SPP is restricted in the neighborhood of dielectric-to-metal interface. Detention in the neighborhood of dielectric interface leads to the strengthening of electromagnetic filed in the interface to create an extremely high sensitivity of SPP for the surfaces.
The antenna is a common bi-directional electrical device which exhibits many structures, shapes and sizes. It converts electric power into electromagnetic waves (transmitting antenna) or receive the electromagnetic waves and converts them into the electric signals (receiving antenna). In the visible range, all usual light emitting sources may be considered as the transmitting antennas. The Maxwell theory of electromagnetic waves states that all frequencies received and transmitted by an antenna are independent and separable, therefore there is no interplay between the frequencies. Thus one may expect the constant efficiency of antenna for each frequency upon which it operates. The operation of today microwave network can serve as an example of this principle, when the signals themselves, i.e. the phone signals, are much weaker than the noises received by the phone antenna. However, this independence of frequencies may not persist when moving into the nanoscale, when the quantum effects dominate. How the usual $\mathrm{GHz}$ antenna will operate when its surfaces are sensitive to plasmonic effect is still a question. As known, the working frequency region of a given antenna depends on its geometrical parameters such as size, shape, and surface structure. The microstrip antenna, for examples, includes a conductive plate on one side of a dielectric plate. The shape of the conductive plates can be square, rectangular, circular, elliptical, or ring. The height of the substrate should be smaller than the operation wavelength, 
but not smaller than $5 \%$ of wavelength. The width $W$ of the antenna controls the input impedance. Larger widths can increase the bandwidth. By increasing the width, the impedance of the feed line can be reduced. A single patch antenna has a typical directivity of $8-9 \mathrm{dBi}$ and a gain approaching $20 \mathrm{dBi}$ [2], [3], [10]. The surfaces of the $\mathrm{GHz}$ antenna are usually too large to be effective for the plasmons to occur under radiation of visible light. Therefore, under normal conditions, there is no change in the efficiency of antenna when it is exposed to the light. The problem may be quite different if its active (conductive) surface consists of small metallic particles of nanoscale size, so that the plasmon resonance happens when exposing to light.

In metallic nanoparticles the optical properties are caused by conduction electrons which oscillate collectively at certain frequency under applied radiation field (visible light). The electron cloud, as conducting medium, possesses a negative (effective) dielectric constant. Our basic idea originates in how a response of a microwave antenna changes when its surface is coated by the suitable metallic nanoparticles that allow the surface plasmon to occur. This antenna may express a better, or a worse response, under radiation of visible light [3], [4]. This consideration may bring important issues as the dielectric constant of electron cloud varies as the plasmon occurs and which should in turn induce a different response of the antenna. The plasmon should affect the impedance characteristics, resonant frequency, bandwidth and performance of antenna [4], [7], [9]. As known, the surface plasmons depend on the size and shape of coated nanoparticles as well as on the nature of used metals and the dielectric properties of surrounding media.

\section{Experimentals}

In our case, the microwave antennas were fabricated using the silica substrates one side coated with gold nanoparticles by mean of Chemical Vapor Deposition (CVD) technique on the Copper $(\mathrm{Cu})$ surface. The surfaces are as shown in Figure 1. This antenna works as a standard microstrip antenna, the following formula for the frequency can be used [3], [4]:

$$
f_{r}=\frac{c}{2 L \sqrt{\varepsilon_{r}}}
$$

where $f_{r}$ is the frequency, $L$ the cell size, $c$ the velocity of light in vacuum and $\varepsilon_{r}$ the relative dielectric constant of glass substrate $\left(\varepsilon_{r}=5\right)$.
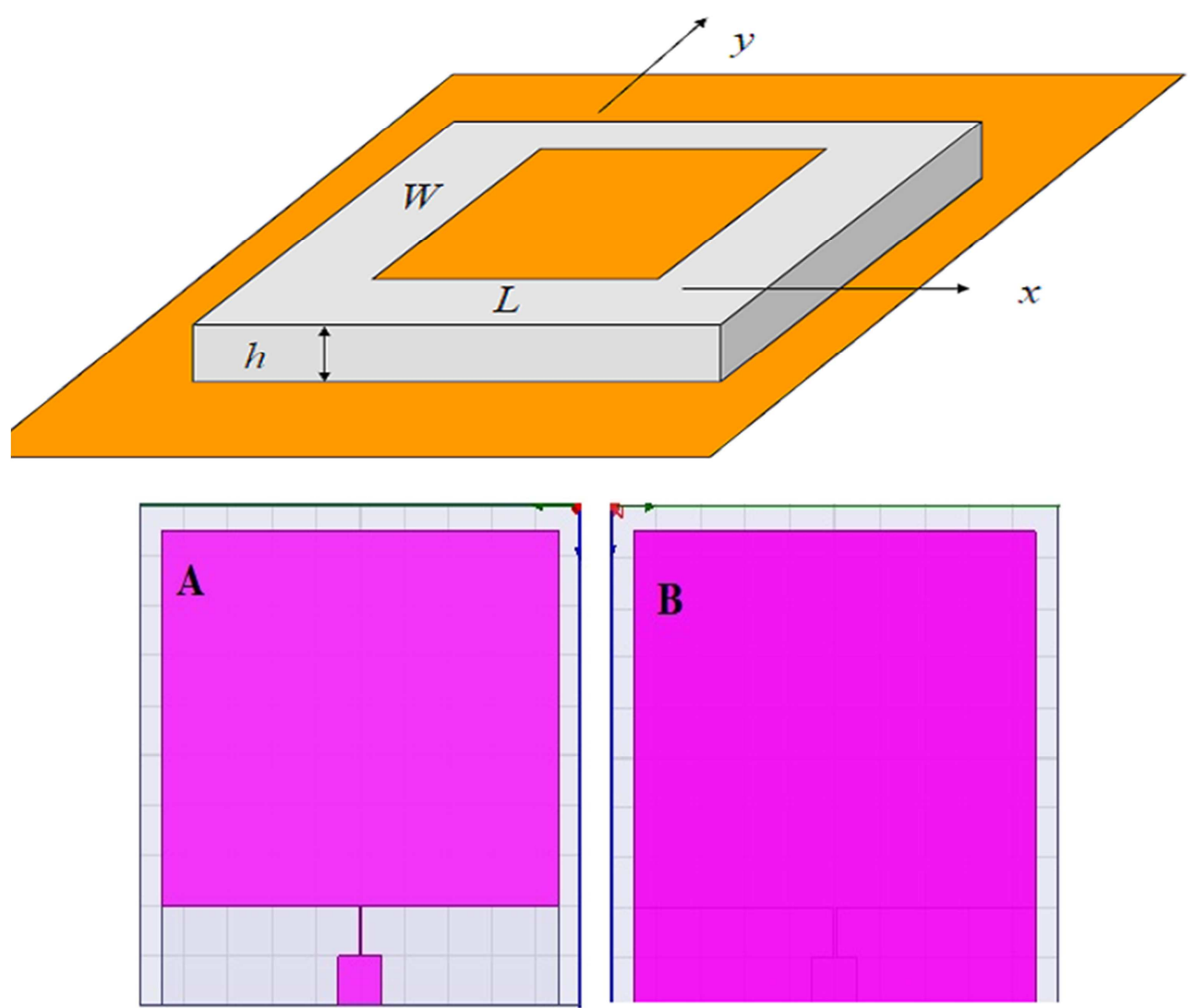

Figure 1. Structure of a microwave antenna: front and back side, $L$ is the length $(10 \mathrm{~mm}), W$ the width $(10 \mathrm{~mm}), \mathrm{h}$ the thickness of the antenna $(1 \mathrm{~mm})$. The active area is $1 \mathrm{~cm}^{2}$.

The surface B is coated with gold $(\mathrm{Au})$ nanopraticles of spherical shape and size from 60 to $100 \mathrm{~nm}$, particularly 60 , 80 and $100 \mathrm{~nm}$. The samples were marked as M1 (60 nm), M2 $(80 \mathrm{~nm})$ and M3 (100 nm). The responses of these sample antennas were studied in the frequency range from 5 to $25 \mathrm{GHz}$ using the frequency analyzer Anritsu 37369D under the radiation of visible light of constant intensity. 


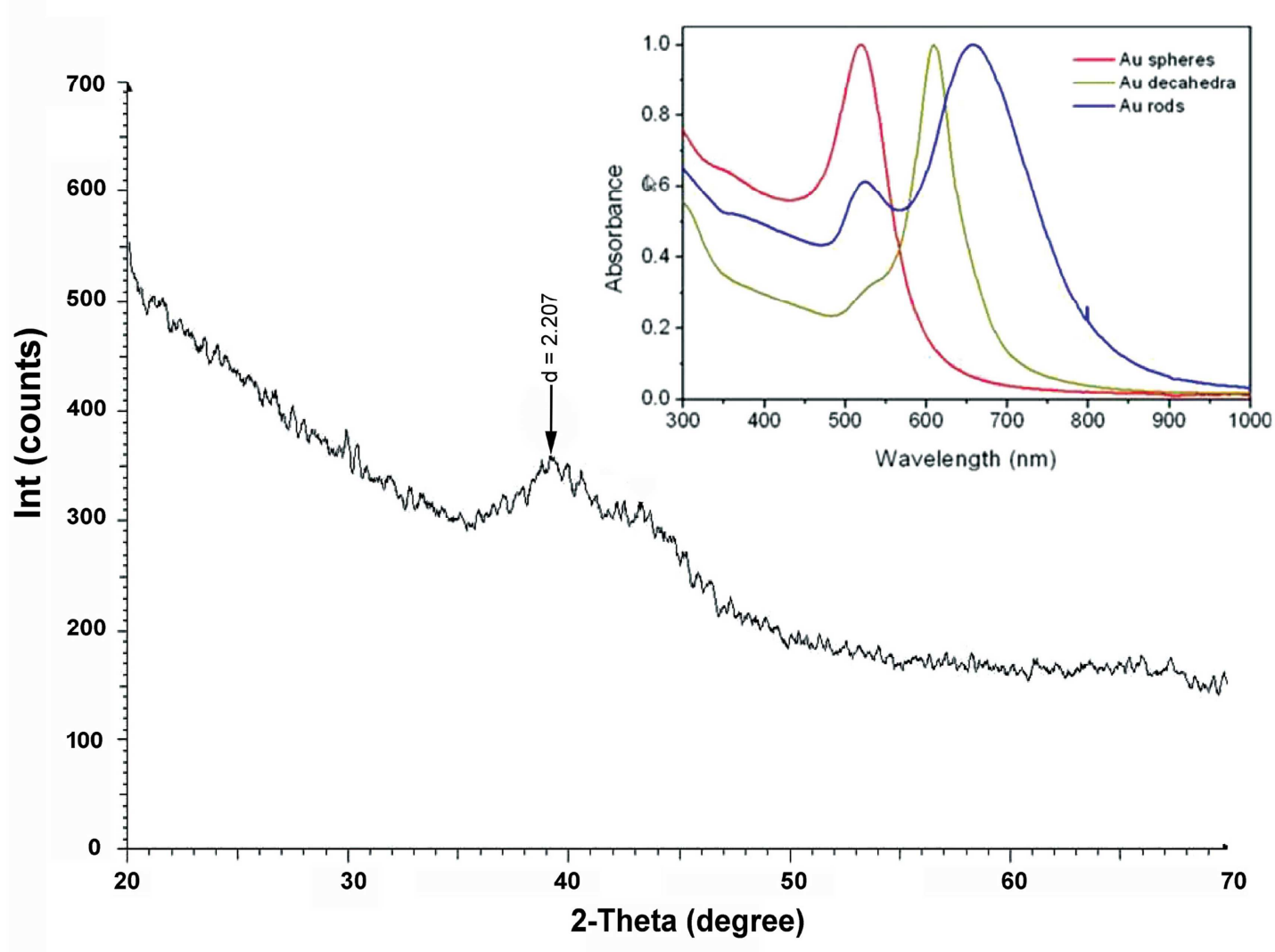

Figure 2. The x-ray scattering diffractogram of coated thin film of gold on the surface of the microstrip antenna, the inset shows the typical absorption of various types of gold nanoparticles.

The gold nanoparticles themselves have been obtained by mean of the seed-mediated method which was introduced by Jana et al. [5]. This method has been later improved by a series of researchers including Nikoobakht et al. [6] (to whom this research follows). By this method the nanoclusters of diameters from 20 to $100 \mathrm{~nm}$ with spherical shape were obtained. The x-ray scattering measurement was performed for the samples and the results are shown in Figure 2. The measurement was taken on a Bruker D5005 diffractometer which was equipped with a $\mathrm{CuK}_{\alpha}$ radiation of wavelength $\lambda$ $=1.54180 \AA$. On the basis of given diffractograms the cell parameter of the crystalline gold was determined to be 4.08 $\AA$. This value agrees well with the cell parameter of the metallic gold reported by various authors previously.

From the typical absorption of gold nanoparticles showed in the inset of Figure 2 we can reveal that absorption of spherical gold nanoparticles centers at around $\lambda=500 \mathrm{~nm}$. The corresponding color pattern for this absorption is lightred. In general, the spherical gold nanoparticles can show the color patterns from yellow to red, ligh-red and even blue. It is noteworthy that the color exactly reflects the change in the effective dielectivity of the electron cloud surrounding the nanoparticles.

\section{Results and Discussion}

Figure 3 shows the responses of the antennas which have the maxima at around $8.5 \mathrm{GHz}$. The measurements were performed in a closed room of area $30 \mathrm{~m}^{2}$ under a constant radiation of 20000 lumens bright LEDs.

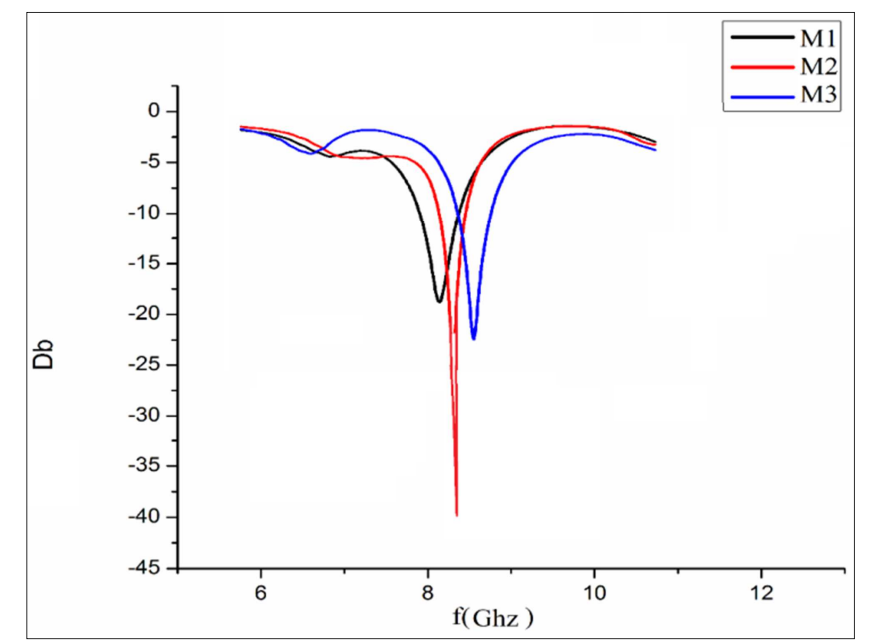

Figure 3. The feedback of the antenna samples when the light was switched off.

As seen, the sample M2, which was coated by $80 \mathrm{~nm}$ gold nanoparticles, showed a twice larger response loss $(-40 \mathrm{~dB})$ in comparison with the ones from the rest two samples. Figure 4 compares the responses of three antennas in the presence and absence of light radiation. 


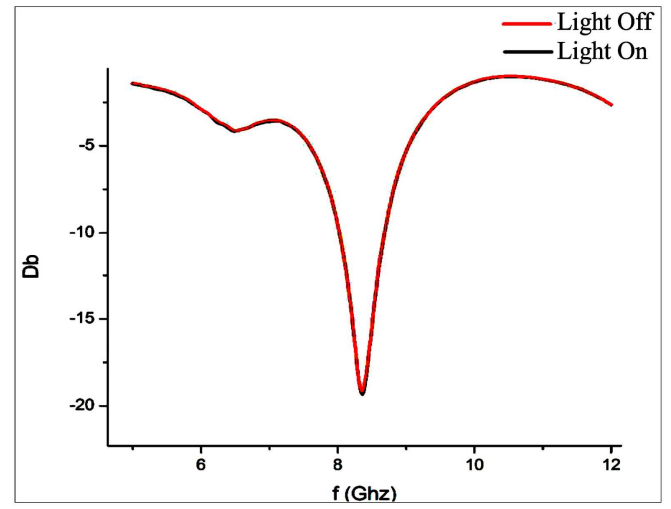

(a) M1

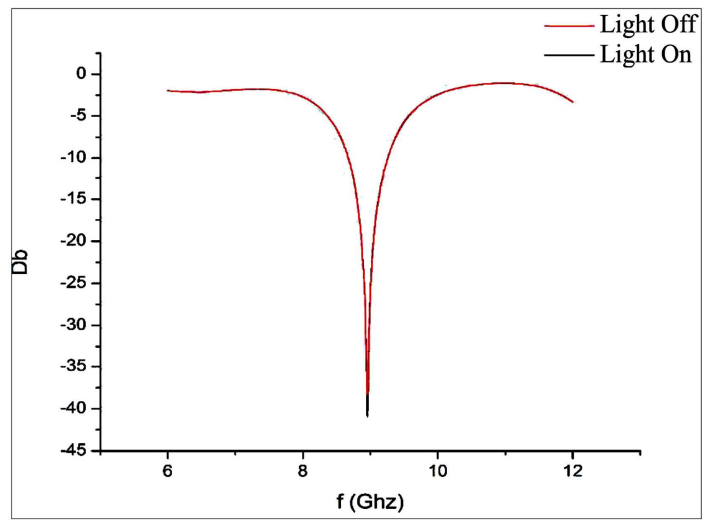

b) M2

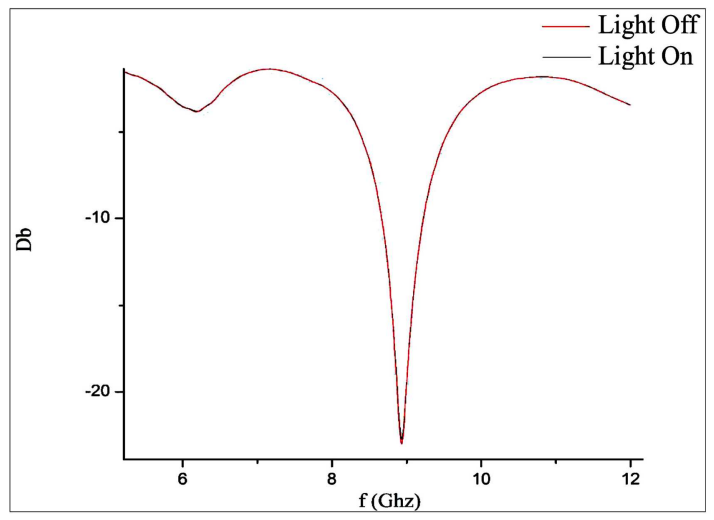

c) M3

Figure 4. The feedbacks of the antennas in the presence and absence of radiation.

The responses were not changed sufficiently as expected, but differences were recognized. Figure 4 shows that when coated with $60 \mathrm{~nm}$ gold nanoparticles (M1) the responses were not significantly different, the response loss (S1 coefficient) changed a little of $0.4 \%$ from -19.15 to -19.24 $\mathrm{dB}$. The electric conductivity of M1 was also measured and the result revealed that M1 conducted electricity very poorly.

With M2, the signals varied about $2.3 \%$ when the light was turned on. And with M3 the signal reached 1.1\% (from 22.7 to $23.0 \mathrm{~dB}$ ). Therefore we conclude that the sample M2 with
$80 \mathrm{~nm}$ nanoparticles coated exhibits a largest response loss. To evaluate the dielectric constants of coated gold surfaces, the complex impedances of the surfaces were measured (by using AUTOLAB/PGSTAT302N equipment). The schematic connection is showed in Figure 5. The antenna works in this case as a RC resonance circuit without an induction coil. From this equivalent circuit we determined the impedance:

$$
\begin{gathered}
Z=R-\mathrm{i} / \mathrm{C} \omega ; \\
Z^{\prime}=\mathrm{R}_{\mathrm{e}}(\mathrm{Z})=\mathrm{R} ; \\
\mathrm{Z}^{\prime \prime}=\mathrm{I}_{\mathrm{m}}(\mathrm{Z})=1 /(2 \pi \mathrm{Cf})
\end{gathered}
$$
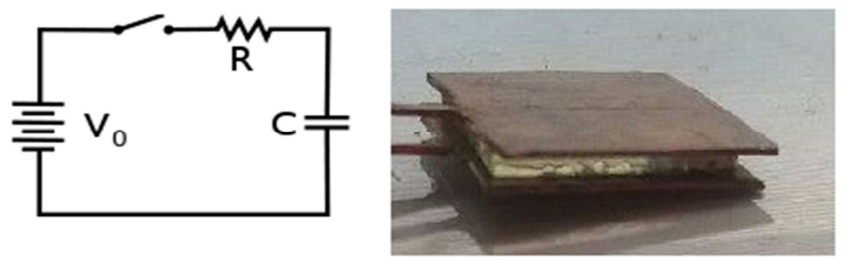

Figure 5. The schematic connection of the antenna and the $R C$ equivalent circuit.

The dependence of impedance on frequency is expressed in Figure 6 for each sample M1 (a), M2 (b) and M3 (c). From these graphs one can reveal that both Z', Z' vary according to the frequency $f$ for all 3 samples. Further, from these data the dependences of $Z$ " on $1 / f$ were determined, where results are shown in Figure 7.

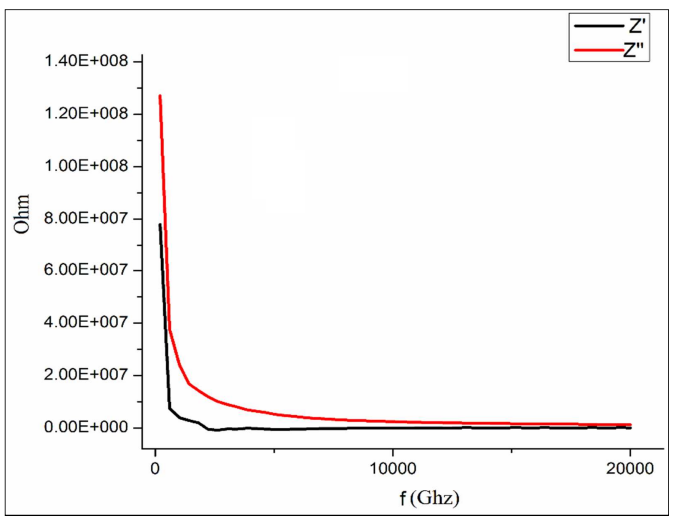

(a) M1

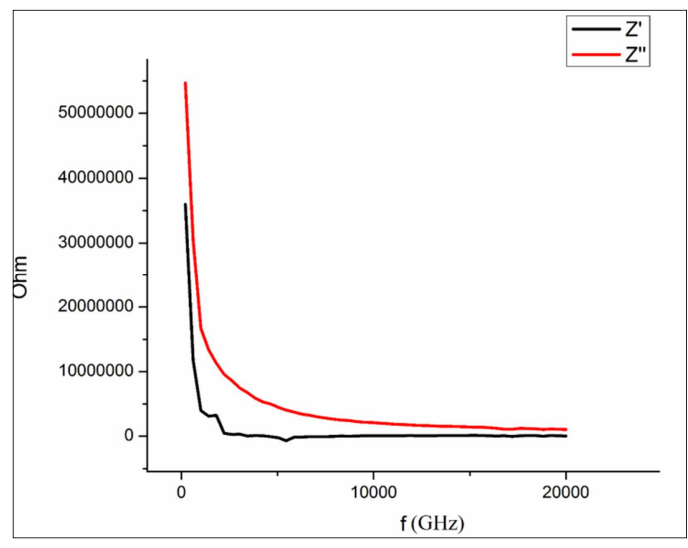

(b) M2 


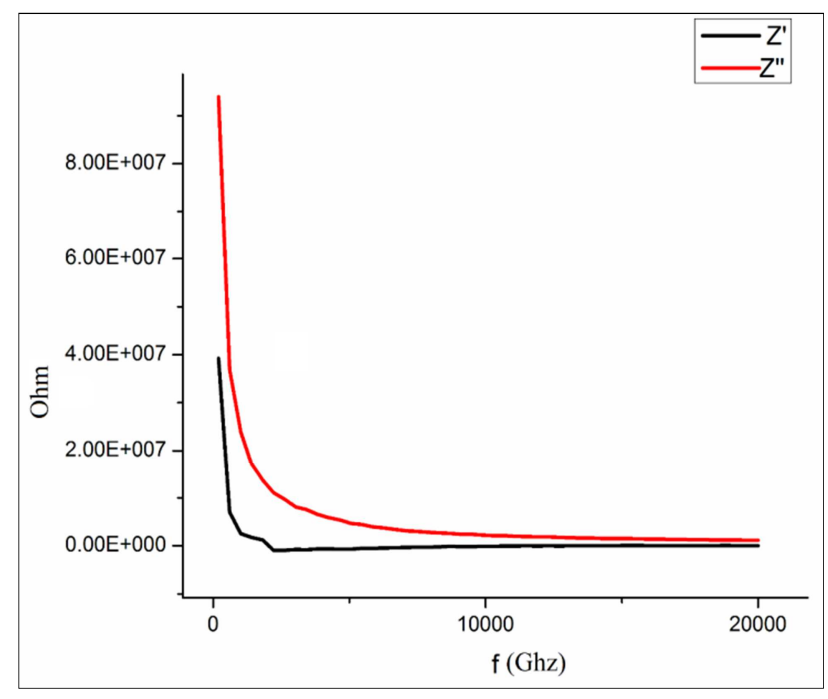

(c) M3

Figure 6. The dependences between $Z$ ' and $Z$ " according to the frequency $f$ for the samples $M 1$ (a), M2 (b) and M3 (c).

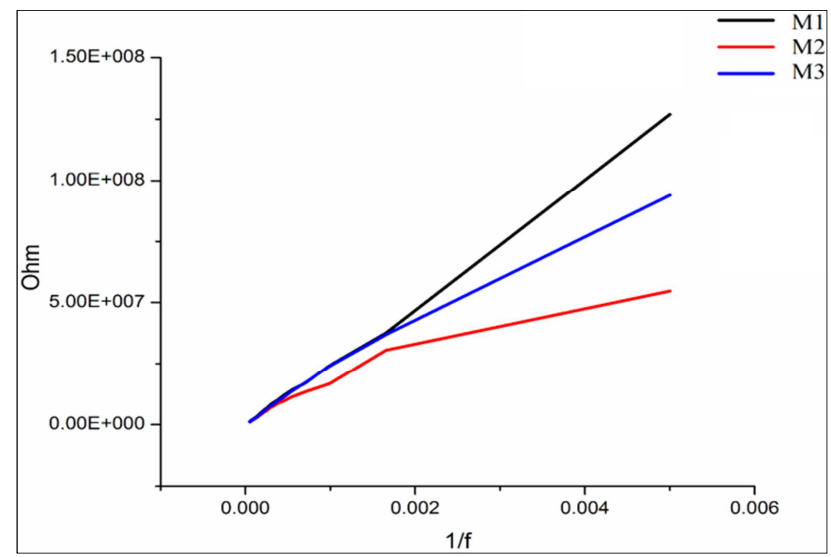

Figure 7. The dependence of Z" on (1/f) for the samples.

On the basis of the relation ( 2 ): $Z$ " $=1 / 2 \pi f C$, the values of capacitances were extrapolated: $\mathrm{C}_{1}=5.8, \mathrm{C}_{2}=6.9$ and $\mathrm{C}_{3}=$ $7.2 \mathrm{pF}$ for M1, M2 and M3 correspondingly. Thus it is clear that the impedance is inversely proportionate to the gold film thickness (the corresponding thicknesses are 60, 80 and $100 \mathrm{~nm}$ ). The thicker were the films the better capacitance value was obtained. Therefore, it is preferable to fabricate the thicker films. We hope this result will help to fabricate the antennas which produce a better response loss (when the light goes on). This will be the plan for the future study.

\section{Conclusion}

This research demonstrates that the microwave antenna possesses the different response losses, when exposed to the visible light radiation, if its surface was coated with the metallic nanoparticles of different thicknesses. The obtained results showed that the antenna operated differently in the presence and absence of visible light radiation, which can be understood by mean of plasmon effects, but to fully understand the effects observed the next study with possibly thicker films need to be performed. At this stage the observed effect seems to be related to the change in the electromagnetic field (dielectric constants too) of the antenna's active surfaces when exposing to light. We leave this for the future consideration.

\section{Acknowledgments}

This research is funded by the Vietnam National University, Hanoi (VNU) under project number QG.15.12

\section{References}

[1] Katherine A. Willets and Richard P. Van Duyne, Localized Surface Plasmon Resonance Spectroscopy and Sensing, Annual Review of Physical Chemistry 58, p. 267 (2007) DOI: 10.1146/annurev.physchem.58.032806.104607.

[2] James JR, Hall PS, Wood C, Peregrinus P. Microstrip Antenna Theory and Design, London, UK,: Peter Peregrinus, (1981).

[3] Balanis, CA; Antenna Theory: Analysis and Design, 3rd Ed., Wiley-Interscience (2012).

[4] Nguyen Van Hai, Nguyen Khac Thuan, Nguyen Duc Tho, and Dang Thi Thanh Thuy, Characterization of a Linear-Structured Meta-Antenna, Journal of Scientific Research \& Reports 4 (1), pp. 28-34 (2015) ISSN: 2320-0227.

[5] N. R. Jana, L. Gearheart, and C. J. Murphy, J. Phys. Chem. 105 B (2001) 4065.

[6] B. Nikoobakht and M. A. El-Sayed, Chem. Mater. 15 (2003) 1957.

[7] A. Tao, S. Habas, and P. Yang, Small 4, p. 310 (2008).

[8] Cao, W.-Q.; Liu, A. J.; Zhang, B.-N.; Yu, T.-B.; Guo, D.-S.; Wei Y.; Qian, Z.-P. Multi-Band Multi-Mode Microstrip Circular Patch Antenna Loaded With Metamaterial Structures. Journal of Electromagnetic Waves and Applications 26 (7), p. 923 (2012).

[9] Eleftheriades, GV; Iyer, A. K.; Kremer, P. C. Planar Negative Refractive Index Media Using Periodically L-C Loaded Transmission Lines. IEEE Transactions on Microwave Theory and Techniques 50 (12), 2702 (2002).

[10] J. Pendry, A. Holden, D. Robbins, and W. Stewart, "Magnetism from conductors and enhanced nonlinear phenomena," Microwave Theory and Techniques, IEEE Transactions on Microwave Theory and Techiniques 47, p. 2075 (1999).

[11] M. L. Brongersma and P. G. Kik, Surface Plasmon Nanophotonics (Berlin: Springer) (1988). 